\title{
Numerical Simulation for Coupled Heat and Moisture Transfer in Building Material
}

\author{
Bing Zhang ${ }^{1, a^{*} \text {, Zhongqing Cheng }}{ }^{2, b}$ and Liang Zhang ${ }^{3, c}$ \\ ${ }^{1}$ Department of Logistic Command and Engineering, Naval University of Engineering, Tianjin,China \\ ${ }^{2}$ Department of Logistic Command and Engineering, Naval University of Engineering, Tianjin,China \\ ${ }^{3}$ Goods and materials purchase department of Beijing, Beijing. China \\ a zhangbing01189@163.com, ${ }^{b}$ bace@tom.com, ${ }^{c}$ zhangliang@tom.com
}

Keywords: Building material; Coupled heat and moisture transfer; Analytical solution; Partial differential equations; Potential function

Abstract. Based on analyzing the mechanism of coupled heat and moisture transfer in building material, this paper takes temperature and moisture potential as driving force. We constructed the model of coupled heat and moisture transfer in building material, solved this model and got the analytical solutions for the distribution of temperature and moisture. Finally this model was tested and verified by using datas from other literature, and a good agreement was obtained. So this method is accurate enough to be used to simulate and forecast the problems of heat and mass transfer in building material.

\section{Introduction}

Most building materials are porous, such as concrete, wood and soil, which are easy to contain moisture. The comprehensive action of temperature and thickness of vapour make moisture transfer very complicated and phase transformation. The accumulation of moisture in building materials will reduce the function of heat insulating material, corrode metalwork, and provide the condition for moulds growing. So it is important to study the mechanism of coupled heat and moisture transfer in building material.

To describe hygrothermal transfer in capillary porous media, Luikov developed a model [1] assuming analogy between moisture migration and heat transfer. On the base of Luikov, the following researchers improved the model and provided corresponding resolvers and results. Mikhailov and Ozisik ${ }^{[2][3]}$ provided resolution based on classical integral transform. Cheroto ${ }^{[4]}$ provided a kind of modified systemic analytic method to get approximate value. Fudym et a ${ }^{[5]}$ solved the one-dimensional non- steady state coupled heat and moisture transfer in building material. Chang et $\mathrm{al}^{6]}$ try to use reduced coupling technique to solve the control equation.

\section{Assumption of model}

According to the research of correlation documents, on the base of weighing and considering the balance of feasibility and precision, the following assumptions are still adopted in this study:

-The material is considered homogenous and the thermophysical properties are assumed constant.

- A local thermodynamic equilibrium between the fluid and the porous matrix is assumed.

- The initial moisture content and temperature repartition in the wall is uniform.

\section{Energy and mass conservation equations}

This study draw lessons from[7], which can satisfy he cases of slab, hollow cylindrical and hollow spherical geometries synchronously.

$$
\rho C_{p} x^{1-2 n} \frac{\partial T}{\partial t}=k \frac{\partial}{\partial x}\left(x^{1-2 n} \frac{\partial T}{\partial x}\right)+\rho C_{m}\left(\varepsilon h_{l v}+\gamma\right) x^{1-2 n} \frac{\partial m}{\partial t}
$$




$$
\rho C_{m} x^{1-2 n} \frac{\partial m}{\partial t}=D_{m} \frac{\partial}{\partial x}\left(x^{1-2 n} \frac{\partial m}{\partial x}\right)+D_{m} \delta \frac{\partial}{\partial x}\left(x^{1-2 n} \frac{\partial T}{\partial x}\right)
$$

where $n=1 / 2$ for slab, $n=0$ for hollow cylinder, and $n=-1 / 2$ for hollow sphere. $T$ is the temperature, and $m$ is the moisture potential, $k$ and $D_{\mathrm{m}}$ are the thermal and moisture conductivity coefficients, respectively, $C_{p}$ and $C_{m}$ are the heat and moisture capacities of the medium, respectively, $\rho$ is the material density, $h_{l v}$ isthe heat of evaporative phase-change, $\gamma$ represents the heat of absorption or desorption, $\delta$ is the thermo gradient coefficient, and $\varepsilon$ is the ratio of the vapor diffusion coefficient to the coefficient of total moisture diffusion. All the material properties mentioned above are effective properties.

In this study, building material is slab.So Eqs.(1) and (2) becomes (3) and (4) ${ }^{[8][9], ~ w h i c h ~ a r e ~}$ structured according to the one-dimensional heat and mass conservation.

$$
\begin{aligned}
& \rho C_{p} \frac{\partial T}{\partial t}=k \frac{\partial^{2} T}{\partial x^{2}}+\rho C_{m}\left(\varepsilon h_{l v}+\gamma\right) \frac{\partial m}{\partial t} \\
& \rho C_{m} \frac{\partial m}{\partial t}=D_{m} \frac{\partial^{2} m}{\partial x^{2}}+D_{m} \delta \frac{\partial^{2} T}{\partial x^{2}}
\end{aligned}
$$

To simplify the notation, Eq. (3) into (4), then rearranging the two new equations, yields

$$
\begin{aligned}
& L \frac{\partial^{2} T}{\partial x^{2}}=\frac{\partial T}{\partial t}-\mu \frac{\partial m}{\partial t} \\
& D \frac{\partial^{2} m}{\partial x^{2}}=\frac{\partial m}{\partial t}-\vartheta \frac{\partial T}{\partial t}
\end{aligned}
$$

where: $\mu=\frac{C_{m}\left(\varepsilon h_{l v}+\gamma\right)}{C_{p}} ; \vartheta=\frac{C_{p} D_{m} \delta}{k C_{m}+C_{m}\left(\varepsilon h_{l v}+\gamma\right) D_{m} \delta} ; L=\frac{k}{\rho C_{p}}$;

$$
D=\frac{k D_{m}}{k \rho C_{m}+\rho C_{m}\left(\varepsilon h_{l v}+\gamma\right) D_{m} \delta}
$$

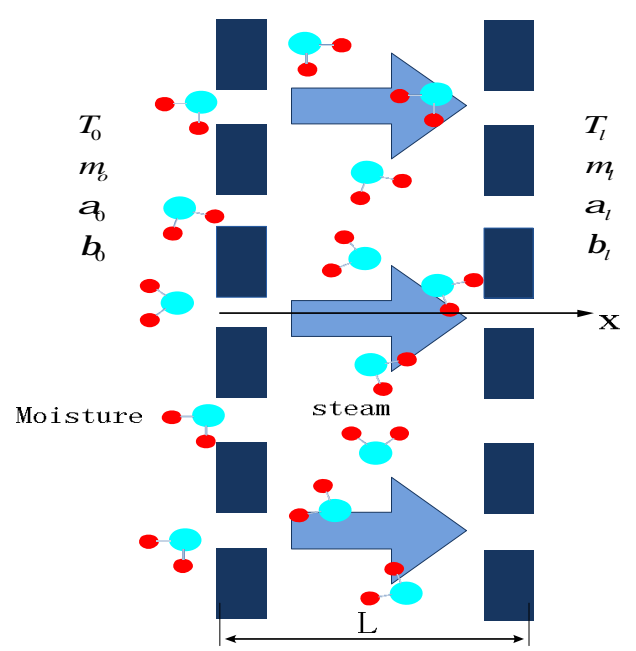

Fig1 Physical model of coupled heat and moisture transfer 


\section{Boundary conditions}

The Physical model of coupled heat and moisture transfer is shown in Fig1, its boundary and initial conditions are:

$$
\begin{aligned}
& \left.k \frac{\partial T(x, t)}{\partial x}\right|_{x=0}=\alpha_{0}\left[T(0, t)-T_{0}\right]+(1-\varepsilon) h_{l v} \beta_{0}\left[m(0, t)-m_{0}\right] \\
& -\left.k \frac{\partial T(x, t)}{\partial x}\right|_{x=l}=\alpha_{l}\left[T(0, t)-T_{l}\right]+(1-\varepsilon) h_{l v} \beta_{l}\left[m(l, t)-m_{l}\right] \\
& \left.D_{m} \delta \frac{\partial T(x, t)}{\partial x}\right|_{x=0}+\left.D_{m} \frac{\partial m(x, t)}{\partial x}\right|_{x=0}=\beta_{0}\left[m(0, t)-m_{0}\right] \\
& -\left.D_{m} \delta \frac{\partial T(x, t)}{\partial x}\right|_{x=l}+\left.D_{m} \frac{\partial m(x, t)}{\partial x}\right|_{x=l}=\beta_{l}\left[m(l, t)-m_{l}\right]
\end{aligned}
$$

initial conditions:

$$
\begin{aligned}
& T(x, 0)=T_{b} \\
& m(x, 0)=m_{b}
\end{aligned}
$$

\section{Solution}

The Laplace transformation of the following groups were introduced from Eq.(7) (12).Introducing transfer function $\phi(x, s)$ :

$$
\begin{array}{r}
\bar{T}(x, s)=\frac{\mu}{L} \phi(x, s)+\frac{T_{b}}{s} \\
\bar{m}(x, s)=\frac{\phi(x, s)}{L}-\frac{1}{s} \frac{d^{2} \phi(x, s)}{d x^{2}}+\frac{m_{b}}{s}
\end{array}
$$

For $x=0: \bar{T}(0, s)=\frac{T_{0}}{s}, \bar{m}(0, s)=\frac{m_{0}}{s}$

For $x=l: \bar{T}(l, s)=\frac{T_{l}}{s}, \quad \bar{m}(l, s)=\frac{m_{l}}{s}$

Taking Eq.(13) and (14) into (5) and (6)中, Eq.(6) becomes:

$$
\frac{d^{4} \phi}{d x^{4}}-\frac{s}{D}\left(1+\frac{D}{L}\right) \frac{d^{2} \phi}{d x^{2}}+\frac{s^{2}(1-\mu \vartheta)}{L D} \phi=0
$$

Solving this Eq.(17) using a rigorous matrix algebra technique, the solution can be written as

$$
\bar{T}(x, s)=\frac{\mu}{L} \sum_{i=1}^{2} \frac{N_{i} \operatorname{sh}\left(q_{i} x \sqrt{s}\right)+M_{i} \operatorname{sh}\left(q_{i}(x-l) \sqrt{s}\right)}{s \cdot \operatorname{sh}\left(q_{i} l \sqrt{s}\right)}+\frac{T_{b}}{s}
$$




$$
\bar{m}(x, s)=\sum_{i=1}^{2}\left[\frac{1}{L}-q_{i}^{2}\right] \frac{N_{i} \operatorname{sh}\left(q_{i} x \sqrt{s}\right)+M_{i} \operatorname{sh}\left(q_{i}(x-l) \sqrt{s}\right)}{s \cdot \operatorname{sh}\left(q_{i} l \sqrt{s}\right)}+\frac{m_{b}}{s}
$$

where

$$
\begin{aligned}
& N_{1}=\frac{\left(m_{l}-m_{b}\right) \mu-\left(1-L q_{2}^{2}\right)\left(T_{l}-T_{b}\right)}{L\left(q_{2}^{2}-q_{1}^{2}\right)}, \quad N_{2}=\frac{\left(1-L q_{1}^{2}\right)\left(T_{l}-T_{b}\right)-\left(m_{l}-m_{b}\right) \mu}{L\left(q_{2}^{2}-q_{1}^{2}\right)}, \\
& M_{1}=\frac{-\left(m_{0}-m_{b}\right) \mu+\left(1-L q_{2}^{2}\right)\left(T_{0}-T_{b}\right)}{L\left(q_{2}^{2}-q_{1}^{2}\right)}, M_{2}=\frac{\left(m_{0}-m_{b}\right) \mu-\left(1-L q_{1}^{2}\right)\left(T_{0}-T_{b}\right)}{L\left(q_{2}^{2}-q_{1}^{2}\right)} 。
\end{aligned}
$$

From [10] their inverse Laplace transformation can be written in the following form:

$$
\underset{\%}{\Omega_{0}^{-1}}\left(\frac{\operatorname{sh}(x \sqrt{s})}{\operatorname{ssh}(l \sqrt{s})}\right)=\frac{x}{l}+\frac{2}{\pi} \sum_{n=1}^{\infty} \frac{(-1)^{n}}{n} e^{-\frac{n^{2} \pi^{2} t}{\left(q_{i}\right)^{2}}} \sin \left(\frac{n \pi x}{l}\right)
$$

Using this transformation to (18) and (19),we can get:

$$
\begin{aligned}
& T(x, t)=\frac{\mu}{L} \sum_{i=1}^{2}\left[N_{i} \frac{x}{l}+\frac{2 N_{i}}{\pi} \sum_{i=1}^{\infty} \frac{(-1)^{n}}{n} e^{\left[\frac{-n^{2} \pi^{2} t}{\left(q_{l}\right)^{2}}\right]} \sin \left(\frac{n \pi x}{l}\right)+\left[\frac{M_{i}(x-l)}{l}\right]+\frac{2 M_{i}}{\pi} \sum_{i=1}^{\infty} \frac{(-1)^{n}}{n} e^{\left[\frac{-n^{2} \pi^{2} t}{\left(q_{i}\right)^{2}}\right]} \sin \left(\frac{n \pi(x-l)}{l}\right)\right]+T_{b} \\
& m(x, t)=\sum_{i=1}^{2}\left(\frac{1}{L}-q_{i}^{2}\right)\left[N_{i} \frac{x}{l}+\frac{2 N_{i}}{\pi} \sum_{i=1}^{\infty} \frac{(-1)^{n}}{n} e^{\left[\frac{-n^{2} \pi^{2} t}{\left(q_{i}\right)^{2}}\right]} \sin \left(\frac{n \pi x}{l}\right)+\left[\frac{M_{i}(x-l)}{l}\right]+\frac{2 M_{i}}{\pi} \sum_{i=1}^{\infty} \frac{(-1)^{n}}{n} e^{\left[\frac{-n^{2} \pi^{2} t}{\left(q_{i} l^{2}\right.}\right]} \sin \left(\frac{n \pi(x-l)}{l}\right)\right]+m_{b}
\end{aligned}
$$

\section{Analysis of a special case}

We take woods as example, the experimental data from [7], correlation physical properties and boundary conditions come from [7]. The temperature and moisture diffusion of three-dimensional can be shown in Fig.2,Fig.3, Fig.4, and Fig.5. Which is fitting well to the data in [7].

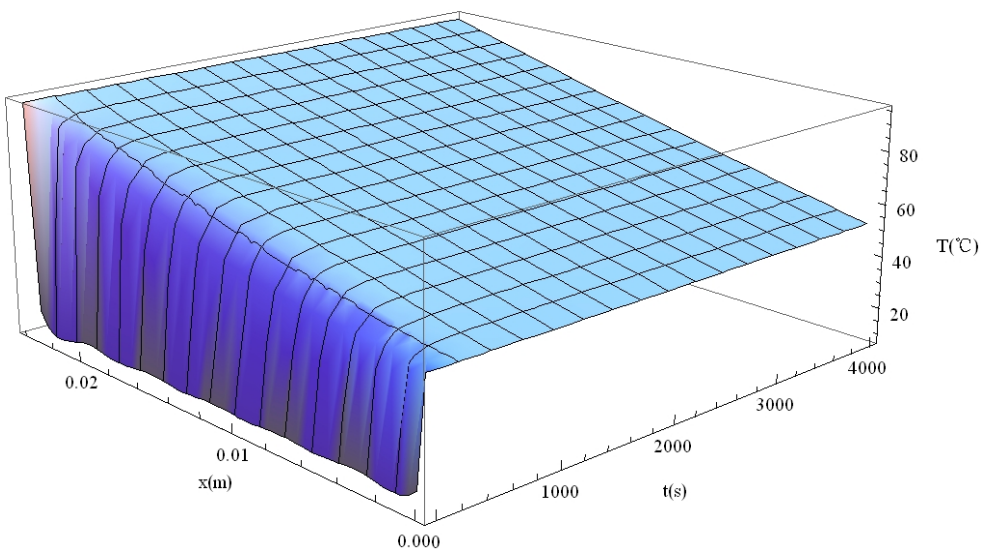

Fig2 Distribution of temperature followed time and space(Case1) 


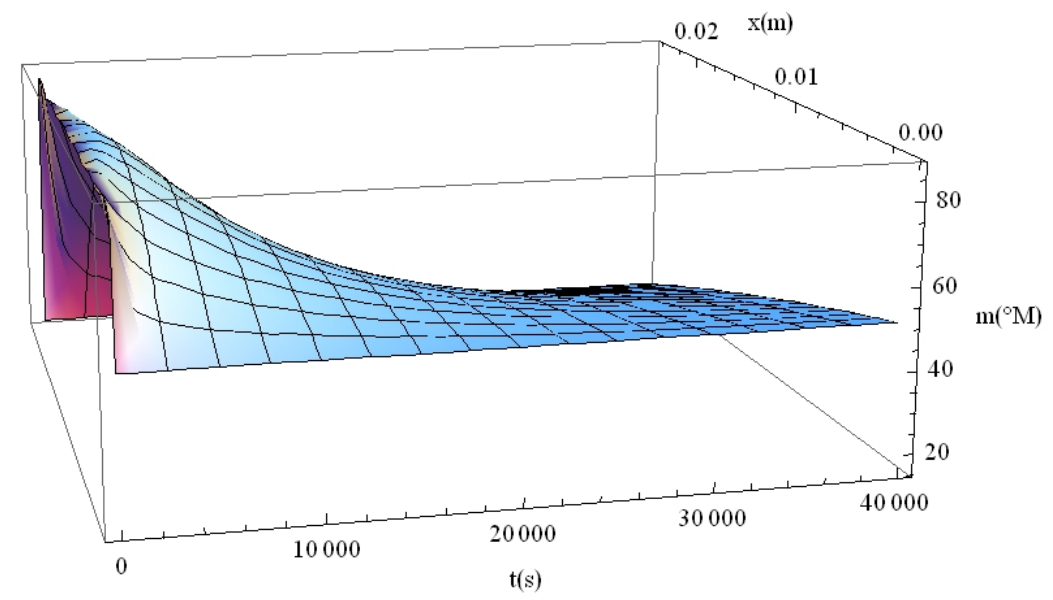

Fig3 Distribution of moisture content followed time and space(Case1)

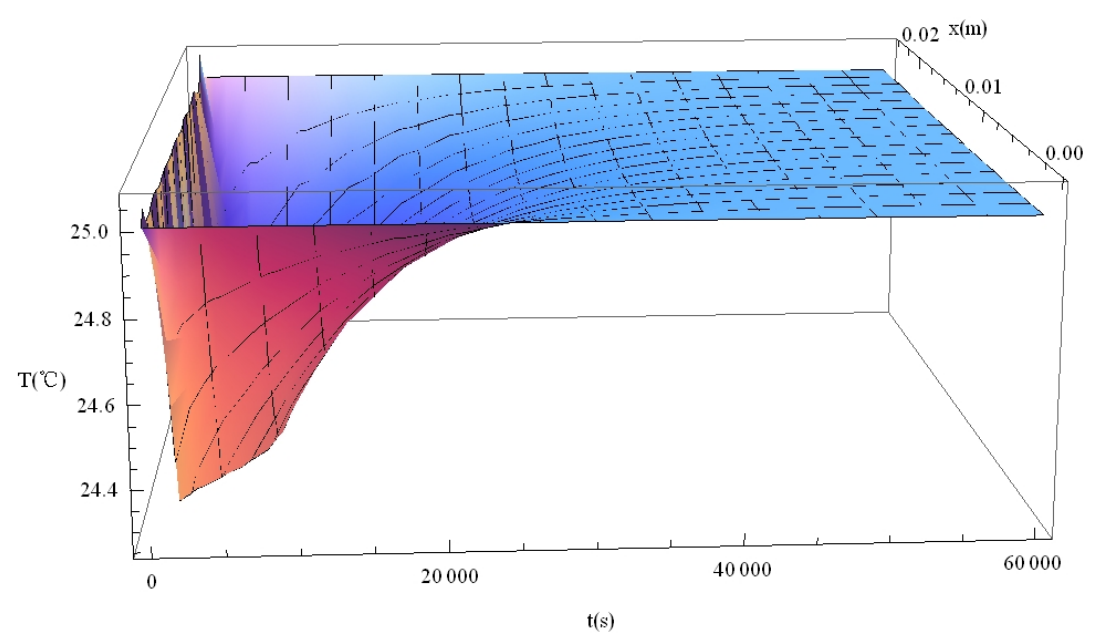

Fig4 Distribution of temperature followed time and space(Case2)

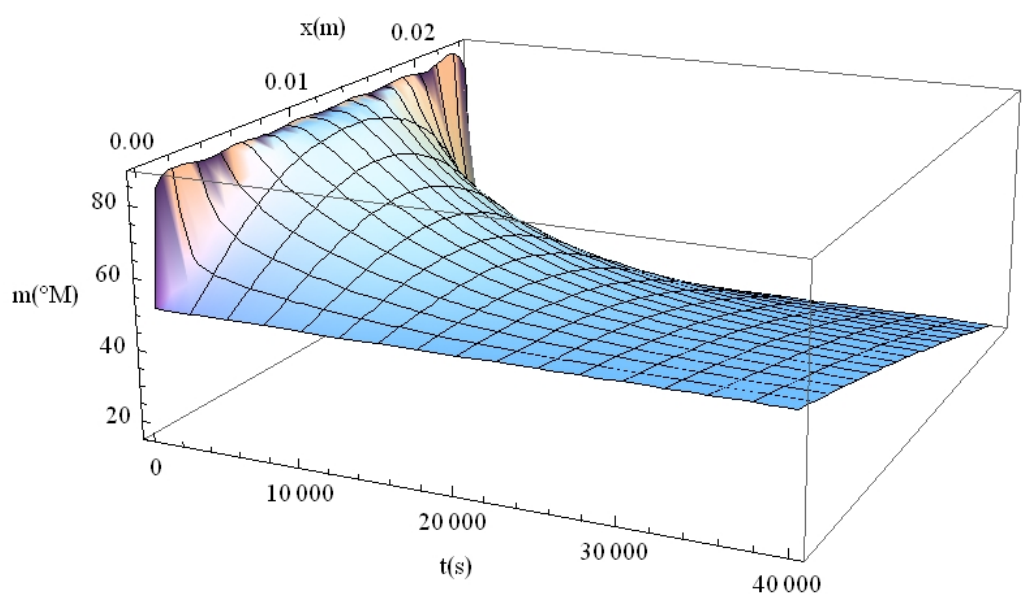

Fig5 Distribution of moisture content followed time and space(Case2)

\section{conclusion}

Based on rational assumption, this paper provides a kind of numerical model to simulate the coupled heat and moisture transfer in building material. This study proposes a new analytical approach that consists of applying the Laplace transform technique and a transformation function to solve the problem of coupled temperature and moisture transport. The results, shown to be the same as 
published analytical solutions obtained using a decoupled technique, compare very well against published analytical solution for a wood slab configuration, and can serve to evaluate the accuracy of approximate or numerical solutions. Therefore, the method is recommended for analytically solving problems involving coupled temperature and moisture transport in porous materials.

\section{References}

[1] Luikov AV. Heat and mass transfer in capillary-porous bodies. Oxford: Pergamon Press, 1966:123-186.

[2] MIKHAILOV M D.OZISIK M N. Unified analysis and solutions of heat and diffusion[M].New York: Wiley, 1984:2-3.

[3] LOBO P D C, MIKHAILOV M D, OZISIK M N. On the complex eigenvalues of Luikov system of equations[J].Dry Technol, 1987, 5(2):273-296.

[4] CHEROTO S, GUIGON S M S, RIBEIRO J W. et al. Lumped-differential formulations for drying in capillary porous media[J].Dry Technol, 1997, 15 (3):811-835.

[5] FUDYM O, BATSALE J C, SANTANDER R. et al. Analytical solution of coupled diffusion equations in semi-infinite media[J].Heat Transfer, 2004,126(3):471-475.

[6] CHANG W J. CHEN T C. WENG C I. Transient hygrothermal stress in an infinitely long annular cylinder: coupling of heat and moisture[J].Thermal Stress. 1991,14 (4):439-454.

[7]Win-Jin Chang,Cheng-I Weng. An analytical solution to coupled heat and moisture diffusion transfer in porous materials[J]. International Journal of Heat and Mass Transfer2000,(43) :3621-3632.

[8] Ribeiro JW. Complete and satisfactory solutions of Luikov equations of heat and moisture transport in a spherical capillary-porous body. International Communications in Heat and Mass Transfer 2000;27:975-984.

[9] Qin M, Belarbi R. Development of an analytical method for simultaneous heat and moisture transfer in building materials utilising transfer function method. Journal of Materials in Civil Engineering ASCE 2005;17(5):492-497.

[10] Spiegel M. Variables complexes (Cours et Problèmes). série Schaum. Mc Graw Hil,1973. 